\title{
梯度折射率超材料透镜
}

\author{
王丹凤, 任致远, 庄国志* \\ 浙江大学航空航天学院, 应用力学研究所, 杭州 310058 \\ *联系人, E-mail: chuangkc@zju.edu.cn \\ 2021-05-31 收稿, 2021-08-25 修回, 2021-08-25 接受, 2021-08-26 网络版发表 \\ 国家自然科学基金(11972318)资助
}

\begin{abstract}
摘要 超材料是由人工设计的、具有天然材料所不具备的超常物理性质的人工复合结构, 有负等效质量密度、负 等效弹性模量、负折射率等特性. 声子晶体超材料是一种具有周期性结构的超材料，其布拉格带隙或局域共振带 隙的存在使处于禁带频率下的声波或弹性波进入声子晶体后无法在其中传播. 其能带特性可以通过设计进行调节, 使通带频率下的声波或弹性波进入具有特定结构的声子晶体后, 实现成像、聚焦以及定向传输等对波传播的极端 控制功能. 梯度折射率超材料是一种折射率随空间变化而变化的结构. 梯度折射率透镜由局部非均匀结构组成, 其 折射率是空间坐标的函数。波在透镜中会沿着弯曲的轨迹传播，可以通过适当的设计实现对波的弯曲、偏转和聚 焦等功能. 梯度折射率的设计可以通过改变局部晶体单元的性质实现有效折射率的梯度分布, 如改变声子晶体的 晶格尺寸、散射体的填充率、散射体的材料等。此外，梯度折射率超材料透镜能够通过设计在宽频率范围内精准 聚焦波, 从而实现能量采集等工程应用. 本文从光学理论原理、透镜设计及其应用三方面展开介绍, 首先对理论基 础以及目前构建梯度折射率器件的几种方法进行总结, 然后从透镜的应用角度介绍几种典型案例, 最后对透镜的 未来研究作出展望.
\end{abstract}

关键词梯度折射率, 透镜, 等效介质, 声子晶体, 超材料

波作为自然界中存在的一种振动传播现象，很早 就被人们所关注. 经典物理学研究领域已经对波的传 播行为进行了大量研究, 包括波参数之间的关系, 波的 反射、干涉、折射现象等. 梯度折射率(graded-index, GRIN)材料是指折射率在空间中逐点变化的非均质材 料 $^{[1]}$. 在梯度折射率材料中传播时, 波沿着弯曲的轨迹 传播. 梯度折射率介质在自然界中很常见, 例如人眼的 晶状体和视网膜受体、地球的大气层等. 许多不寻常 的光学现象(如海市傆楼)就是光线在梯度率介质中发 生弯曲而产生的. 通过设计, 满足特定折射率曲线的梯 度折射率器件可以像传统光学元件(如棱镜、透镜等) 一样, 实现对光的弯曲、汇聚和偏折等功能 ${ }^{[2 \sim 4]}$. 梯度 折射率透镜最早在光学系统中被研究，在克服了早期
材料制造的困难之后, 很大程度上促进了光通信系 统、集成光学以及微光学的发展. 基于光学梯度透镜 的启发, 近 20 年来, 光学梯度透镜的设计理念和功能被 应用于声学 ${ }^{[5 \sim 11]}$ 、水声 ${ }^{[12]}$ 、弹性波 ${ }^{[13 \sim 18]}$ 等领域.

随着近几十年来超材料概念的提出，对波的调控 达到了一个新的高度. 超材料是一种人工构造的具有 超常物理性质的人工复合结构，通过超材料来调控波 的传播可以达到预期的效果. 1987年, John ${ }^{[19]}$ 和Yablonovitch ${ }^{[20]}$ 在研究光在周期性电介质结构中的传播后, 分别提出了光子晶体的概念：当光在人工周期性电介 质材料中传播时，在一定的频率范围内会受到材料的 散射和抑制, 人射波将不能通过光子晶体, 从而产生类 似于半导体禁带的光子带隙. 通过在光子晶体的周期

引用格式: 王丹凤, 任致远, 庄国志. 梯度折射率超材料透镜. 科学通报, 2022, 67: 1279-1289 Wang D F, Ren Z Y, Chuang K C. A review of gradient index metamaterials lenses (in Chinese). Chin Sci Bull, 2022, 67: 1279-1289, doi: 10.1360/TB-20210523 
性结构中引入缺陷，可使处于禁带范围内的光波沿着 缺陷传播, 从而达到人为控制光波传播路径的目的. 与 光子晶体的概念类似, 通过类比光子晶体及周期性弹 性复合材料的性质，散射声子晶体(phononic crystal, PC)的概念于1993年被提出 ${ }^{[21,22]}$. 如图1所示, 禁带频率 下的声波或弹性波进人声子晶体后无法传播. 此外, 由 于周期性散射声子晶体基于布拉格散射理论，其晶格 尺寸需与波长处于同一数量级才能有很好的禁带效果, 在实际工程应用中受限. 因此, Zhu等人 ${ }^{[23]}$ 于 2000 年提 出了基于局域共振理论的声子晶体，提供了产生低频 带隙的思路. 通过设计调节声子晶体的能带特性, 通带 频率下的声波或弹性波进人具有特定结构的声子晶体 后，可以实现成像、聚焦以及定向传输等对波传播的 控制功能 ${ }^{[24-26]}$, 在减振降噪、波导、工业无损检测、

医疗检查等领域具有广阔的应用前景.

常见的用来实现梯度透镜的方式有两种：一种是 借助超材料或超表面 ${ }^{[27,28]}$, 利用人工材料特性或者特 殊的表面处理来实现波的操控效果; 另一种是构造声 子晶体透镜, 通过改变声子晶体的材料属性及几何结 构, 使其能带结构逐渐变化, 进而实现透镜折射率的梯 度分布. 声波传人梯度折射率声子晶体后，由于介质间 折射率的差异, 其传播路径将变得弯曲, 从而实现声波 的弯曲、偏转和聚焦等功能 ${ }^{[29,30]}$.

梯度折射率超材料透镜的实现方式和应用众多，

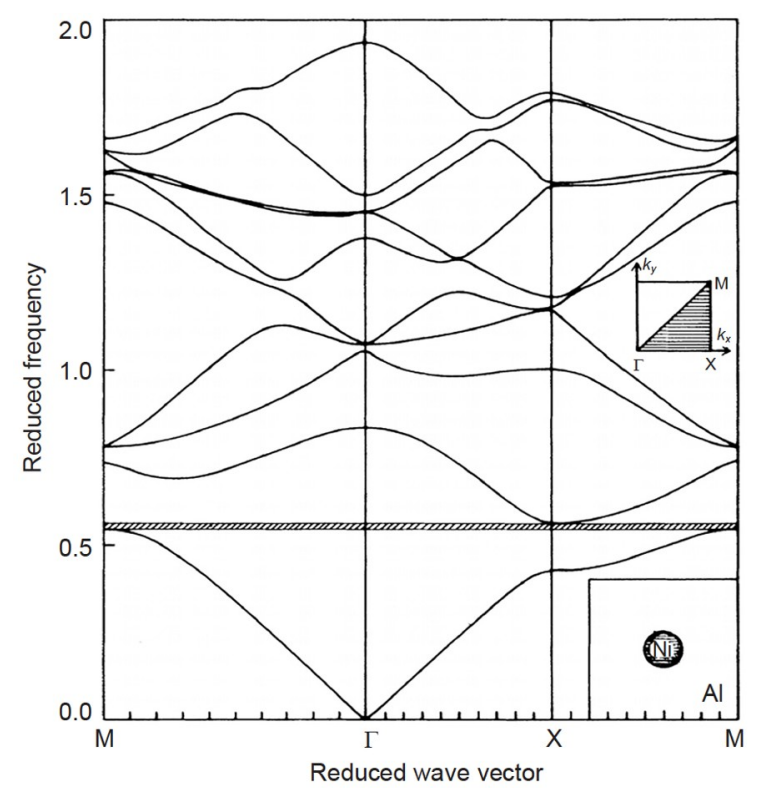

图 1 布拉格散射型声子晶体的能带结构 ${ }^{[21]}$

Figure 1 Band structures of Bragg scattering phononic crystals ${ }^{[21]}$
本文从梯度折射率光学透镜的发展历史、原理和设计 理念出发, 对梯度折射率光学透镜、声学透镜和弹性 波透镜等研究现状进行总结, 回顾了梯度透镜的设计 与波轨迹的关系，对不同的实现方式以及应用形式作 了归纳, 提供了一些有助于后续研究的结论与展望.

\section{1 梯度折射率光学透镜的研究}

\section{1 光学透镜理论研究进展}

在传统光学系统中，每个光学元件的折射率被认 为是固定的, 也就是说, 结构内部的材料参数是常数. 在透镜系统的设计中, 透镜设计者独立地改变每个组 件的曲率、厚度和折射率, 以优化透镜系统的性能. 梯 度折射率透镜最早于1854年提出, Maxwell ${ }^{[31]}$ 设计了一 种被称为Maxwell鱼眼的梯度折射率透镜. 该透镜具有 球面对称的特点, 光在透镜内部聚焦在共轭点上, 在透 镜空间的每一点都能在其共轭点上无散射地成像. 该 透镜的提出在理论上有着极大的意义，而将透镜理论 应用于实际却经过了漫长的技术研究阶段. 在之后的 几十年, 人们研究了各种制造梯度折射率材料的方法: 离子扩散技术 ${ }^{[32]}$ 、离子填充法 ${ }^{[33]}$ 和溶胶-凝胶法 ${ }^{[34]}$ 等. 1905年，Wood ${ }^{[35]}$ 用浸渍技术首次构造了一个折射率轴 对称、对光有汇聚或发散作用的明胶圆柱体，标志着 梯度折射率光学透镜的研究从理论进人了实践.

在之后的几十年中，学者进行了很多梯度折射率 透镜的理论计算与实践研究工作. 1964年, Luneburg ${ }^{[36]}$ 设计了任意平行光束汇聚一点的经典Luneburg透镜, 这一透镜后被推广应用于更复杂的环境 ${ }^{[37,38]}$. 离子扩 散技术的成熟使透镜的制作工艺取得突破，促进了梯 度折射率光学透镜的实践应用 ${ }^{[39,40]}$. 之后, 学者又研究 了光束在空间横向分布梯度折射率介质中的波导效 应 $^{[41]}$ 和类透镜效应 ${ }^{[42]}$, 以及高斯光波在梯度折射率介 质中的轴与离轴传输现象 ${ }^{[43]}$ 等. 此外, Eaton透镜 ${ }^{[44]}$ 、 测地线透镜等的提出也为光学透镜的应用提供了新的 思路. 之后, 梯度折射率透镜被广泛应用于光纤通 信 ${ }^{[4 \sim 47]}$ 、非接触成像 ${ }^{[48 \sim 52]}$ 等工程领域.

\section{2 梯度折射率透镜的理论原理}

按照梯度折射率透镜折射率的空间分布与波传播 轨迹之间的关系, 可以分为径向梯度分布、轴向梯度 分布、层状梯度分布以及球状梯度分布，其中被广泛 应用的是径向梯度分布透镜. 在此, 采用柱坐标系分析 
波在径向梯度透镜中传播的轨迹方程，透镜在 $r \theta$ 平面 存在梯度分布的折射率，波沿着 $z$ 轴传播，其光线方 程为

$\frac{\mathrm{d}}{\mathrm{d} s}\left(n \frac{\mathrm{d} r}{\mathrm{~d} z}\right)=\nabla n$,

其中的折射率分布为

$$
\begin{aligned}
n^{2}(r)= & n^{2}(0)\left(1-h_{2}(\beta r)^{2}+h_{4}(\beta r)^{4}+h_{6}(\beta r)^{6}+\cdots\right. \\
& \left.+h_{2 i}(\beta r)^{2 i}\right),
\end{aligned}
$$

式中, $\beta$ 为折射率分布常量, $h_{2 i}$ 为二次项分布系数.

这里很难根据方程(2)直接求出具体的解析解，但 是针对特殊的折射率分布, 可以求解波轨迹方程. 其中 应用广泛的是满足双曲正割分布的梯度折射透镜，其 折射率分布为(图2(a))

$n=n(0) \operatorname{sech}(\alpha r)$,

相应的波轨迹方程为

$z(r)=\frac{1}{\alpha} \sinh ^{-1}\left(u_{0} \cos (\alpha r)+\dot{u}_{0} \sinh (\alpha r) / \alpha\right)$,

其中, $\alpha$ 表示梯度系数,

$u_{0}=\sinh \left(\alpha z_{0}\right)$.

因此，波在透镜中传播可以周期性地聚焦于一点 (图2(b)), 空间周期为 $f=\pi / 2 \alpha^{[1]}$. 除了被广泛应用的双 曲正割梯度透镜，常见的透镜还有上述的Maxwell鱼眼 透镜、Luneburg透镜等 ${ }^{[53]}$. 不同透镜对应的折射率分 布总结于表1.

\section{2 梯度折射率声子晶体透镜的设计}

光学梯度透镜的设计理论启发了声学透镜和弹性 波透镜的研究，而具有特定折射率变化的梯度材料难 以从自然界直接获取. 在光学中, 人们通过提高镜片制 作工艺来获取梯度折射率透镜. 在声学、弹性波领域,
表 1 几种典型透镜的折射率

Table 1 Refractive indices of several typical lenses

\begin{tabular}{cc}
\hline 透镜 & 折射率 \\
\hline Maxwell鱼眼 & $n(r)=\frac{2}{1+r^{2}}$ \\
Luneburg & $n(r)=\sqrt{2-r^{2}}$ \\
$90^{\circ}$ 旋转 & $n^{4}(r)-2 n(r)+r=0$ \\
Eaton & $n(r)=\sqrt{\frac{2}{r}-1}$ \\
聚焦 & $n(r)=\frac{1}{r}$ \\
\hline
\end{tabular}

可以通过设计包含特殊散射体阵列的人工复合结构来 构造人工梯度材料. 由于散射体的存在, 可以有效改变 声波、弹性波在其中的传播速度. 通过调整散射体的 分布来控制有效速度分布, 可以实现透镜的功能. 散射 体大小、晶格间距的变化等几何参数可以改变有效折 射率. 除了几何参数外, 有效折射率也可以根据散射体 的力学特性进行调整, 例如材料的选择 ${ }^{[8,54]}$ 或者外部刺 激, 如电场 ${ }^{[5]}$ 、温度 ${ }^{[56]}$ 等. 梯度折射率器件的有效折射 率可以小于或大于基体介质的有效折射率，这与波传 播过程中的相位提前 ${ }^{[12]}$ 或延后相对应. 有效折射率的 计算一般有以下方式：基于等效介质理论、基于声子 晶体的能带特性、基于弹性板尺寸空间分布以及变换 介质理论等. 基于变换介质理论是基于坐标变换的方 法，利用超材料使波在现实空间中按照虚拟空间中的 方式传播 ${ }^{[57]}$, 其涉及坐标变换矩阵的代人和推导, 这里 对其实现透镜的方法不展开介绍.

\section{1 基于等效介质理论的声学透镜}

有效速度的计算是通过等效介质理论的数学工具 完成的. 等效介质理论指在等价和近似的基础上研究 复合材料的宏观特性, 在某些特定的研究频率范围, 波
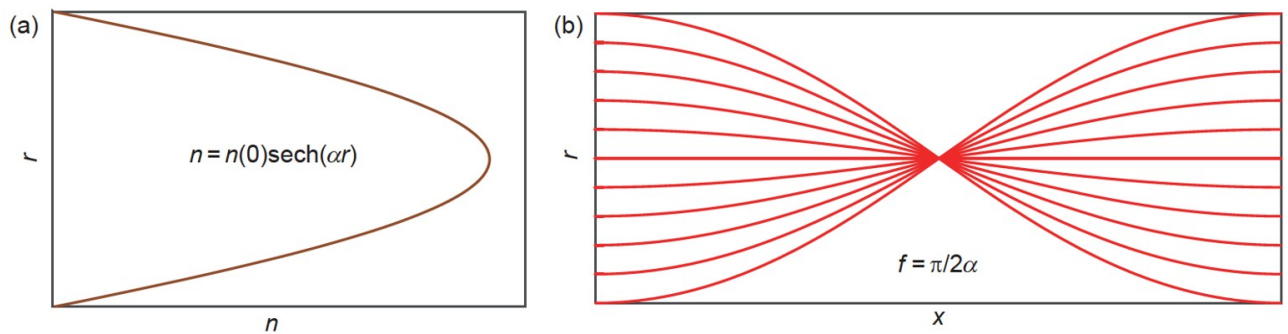

图 2 (网络版彩色)双曲正割折射率透镜. (a) 折射率分布; (b) 梯度折射率透镜的波轨迹

Figure 2 (Color online) Hyperbolic secant refractive index lens. (a) Refractive index profile; (b) ray trajectory of the gradient index lens 
场不区分基体和填充物，而是将结构视为具有某些有 效参数(刚度、密度、黏度等)的均质材料 ${ }^{[58]}$. 例如, 二 维流体散射体(下标为 $\mathrm{a}$ )和流体基体(下标为 $b$ )组成复合 材料, 其等效体积模量和等效质量密度由下式给出:

$\frac{1}{B_{\text {eff }}}=\frac{f}{B_{\mathrm{a}}}+\frac{1-f}{B_{\mathrm{b}}}$,

$\frac{\rho_{\text {eff }}}{\rho_{\mathrm{b}}}=\frac{\rho_{\mathrm{a}}(\Delta+f)+\rho_{\mathrm{b}}(\Delta-f)}{\rho_{\mathrm{a}}(\Delta-f)+\rho_{\mathrm{b}}(\Delta+f)}$,

其中, $f$ 是晶体填充率(填充物面积除以晶胞面积), $\Delta$ 表 示由于多次散射过程而导致填充分数的改变 ${ }^{[59,60]}$.

以声波为例, 声波在空气中传播时, 几乎所有固体 材料的密度都比空气高得多, 而极端的阻抗失配会阻 碍声波穿透固体填充物. 因此, 这些固体可被假定为空 气基体中的“刚性”填充物，其质量密度和可压缩性可 设为无穷大. 此时, 如果填充分数很小, 且可以忽略多 次散射相互作用 $(\Delta$ 可视为 1$)$, 则有效质量密度和速度可 简化为 ${ }^{[59 \sim 62]}$

$\rho_{\mathrm{eff}}=\frac{\rho_{\mathrm{air}}(1+f)}{1-f}$,

$c_{\mathrm{eff}}=\frac{c_{\mathrm{air}}}{\sqrt{1+f}}$.

这种等效介质理论可以推广到更复杂填充散射体 的晶胞计算中, 以计算人工构造的包含散射体晶胞中 的波速. 当声波穿过在空气中的刚性圆柱体时, 可以看 作穿过不同填充尺寸的单元, 而在不同填充尺寸下, 晶 胞具有不同的折射率. 通过合理的尺寸分布或填充不 同材料, 可以设计梯度折射率声子晶体透镜 ${ }^{[5,13,63 \sim 66]}$.

根据基质或填充物的性质，可以将透镜的设计分 为流体或固体两种类型. 在流体情况下, 横波在波场中 不存在, 声场完全由一个标量压力场描述; 而在固体情 况下，必须考虑不同传播速度的横波. 然而，对于流体 填充物, 可以作一个额外的划分, 因为如果背景是空气 或水, 散射过程中涉及的物理机理是不同的. 在空气中, 由于空气与任何固体之间的阻抗不匹配，几乎所有的 固体材料都具有声刚性; 而在水声中, 这种阻抗不匹配 的顺序是相同的, 因此需要考虑填充物的弹性特性. 用 于声波的梯度折射器件遵循1.2节梯度折射率透镜的设 计原则, 一般考虑二维平面透镜. 如空气中声波聚焦的 器件, 可以设计不同半径的金属杆作为散射体, 其折射 率沿着波的传播方向保持不变，在垂直于波传播方向 呈双曲正割分布 ${ }^{[8]}$, 通过计算声波轨迹所满足的微分
方程可以得到焦点位置 ${ }^{[63]}$. 类似地, 针对水声超材料, 学者也提出了类似钢柱散射体分布的透镜设计，考虑 阻抗匹配的因素计算等效参数，可以得到同样的聚焦 现象 ${ }^{[54]}$.

在各向异性超材料中，波具有特殊的传输特性 ${ }^{[67]}$, 可以解决消逝波的衰减问题，进而突破传统透镜的衍 射极限实现亚波长成像 ${ }^{[68]}$. 此外, 具有特殊参数分布的 各向异性超材料可以设计偏振不敏感光学透镜 ${ }^{[69]}$, 通 过调整人射光束的位置可以使相平面上的相位完全重 构. 此类材料可以用来设计光束偏振分离器 ${ }^{[70]}$.

\section{2 基于声子晶体能带特性的透镜设计}

声子晶体透镜主要通过构造声子晶体，利用其能 带特性完成. 按照波传播方式的差异可分为体波声子 晶体、表面波声子晶体和兰姆波声子晶体. 体波声子 晶体的模式和种类简单，上述刚性柱嵌人空气基体的 模型就被广泛研究. Qiu等人 ${ }^{[71]}$ 研究了空气中由六边形 钢柱阵列组成的二维声波晶体中声波的负折射行为和 成像效应，利用负群速度色散带特性实现了负折射聚 焦现象，并在此基础上实现了点源的远程成像. 此外, 表面波声子晶体和兰姆波声子晶体的特殊声禁带特性 可同样被用来设计梯度折射率声子晶体透镜 ${ }^{[8,12,72 ~ 75]}$.

声子晶体透镜的设计一般需要借助声子晶体的能 带特性与等效折射率的关系 ${ }^{[8]}$ :

$n_{\text {eff }}=\frac{n_{\Gamma \mathrm{X}}+n_{\Gamma \mathrm{M}}}{2}$,

$n_{\Gamma \mathrm{X}}=\frac{c_{\mathrm{b}}}{c_{\Gamma \mathrm{X}}}=\frac{c_{\mathrm{b}}}{\mathrm{d} \omega / \mathrm{d}_{\Gamma \mathrm{X}}}$,

$n_{\Gamma \mathrm{X}}=\frac{c_{\mathrm{b}}}{c_{\Gamma \mathrm{M}}}=\frac{c_{\mathrm{b}}}{\mathrm{d} \omega / \mathrm{d}_{\Gamma \mathrm{M}}}$,

其中, $c_{\mathrm{b}}$ 为基体材料中的声速, $c_{\Gamma \mathrm{X}}$ 和 $c_{\Gamma \mathrm{M}}$ 为体弹性波模 态的群速度, $\Gamma \mathrm{X}$ 和 $\Gamma \mathrm{M}$ 为第一布里渊区的两个方向.

对于环氧基声子晶体，剪切-垂直(SV)模态的第一 波段图可以用两种方式绘制：(1) 如图3(a)所示的不同 填充分数(不同半径)的钢填充物; (2) 如图3(b)所示的固 定填充分数的不同材料填充物，不同固体材料的弹性 参数不同. 这两种方法都可以通过式(11)改变第一 SV 模态的斜率, 从而导致有效折射率的变化, 实现波的聚 焦效果(图3(c)). 类似地, Tan等人 ${ }^{[72,73]}$ 采用旋转三瓣形 散射体设计声子晶体透镜，通过计算声子晶体的第一 和第二能带, 得到散射体的旋转角度与声子晶体能带 之间线性变化的关系; 再通过计算能带的切线斜率, 即 
可得到声子晶体沿着波传播方向的群速度，进而求得 相应的折射率. 同时, 利用两条能带对应于等效正折射 率和等效负折射率这一性质，分别设计了梯度正折射 率声子晶体透镜和梯度负折射率声子晶体透镜. 同样 地，Allam等人 ${ }^{[74]}$ 结合3D打印技术，分析了不同填充分 数下的频散曲线, 以设计适用于声波聚焦的装置.

\section{3 基于弹性板尺寸空间分布的透镜设计}

光学透镜和声学透镜启发了板波控制方面的研究. 对于二维薄板或薄膜，弹性波由于板的两个边界的约 束, 产生反对称、对称和剪切水平偏振模态. 在这些模 态中, 由于低阶反对称模态(即弯曲模态)的主要变形特 征是板的面外位移分量, 易于激励和检测，因此得到了 广泛的研究. 除了弯曲模态外, 其他的板波模态 $\left(\mathrm{S}_{0}\right.$ 、 $\mathrm{SH}_{0}$ 等)也被用来设计透镜 ${ }^{[76]}$. 这些利用梯度透镜控制 板波的设计大多遵循上述两种方法.

除了上述通过在基体中添加不同基质改变填充参 数来构造梯度折射率透镜的方式外，板波的控制还可 以利用板自身的参数来实现. 其中, 板中波的色散关系 与相速度方程为

$k^{4}=\frac{\rho h \omega^{2}}{D}$,

$c^{4}=\left(\frac{\omega}{k}\right)^{4}=\omega^{2} \frac{E h^{3}}{12\left(1-v^{2}\right) \rho h}$.

其中, $k$ 为波数, $\rho$ 和 $E$ 分别为板的密度和杨氏模量, $v$ 为 泊松比, $h$ 为板厚, $\omega$ 为频率, $c$ 为板波传播的相速度.
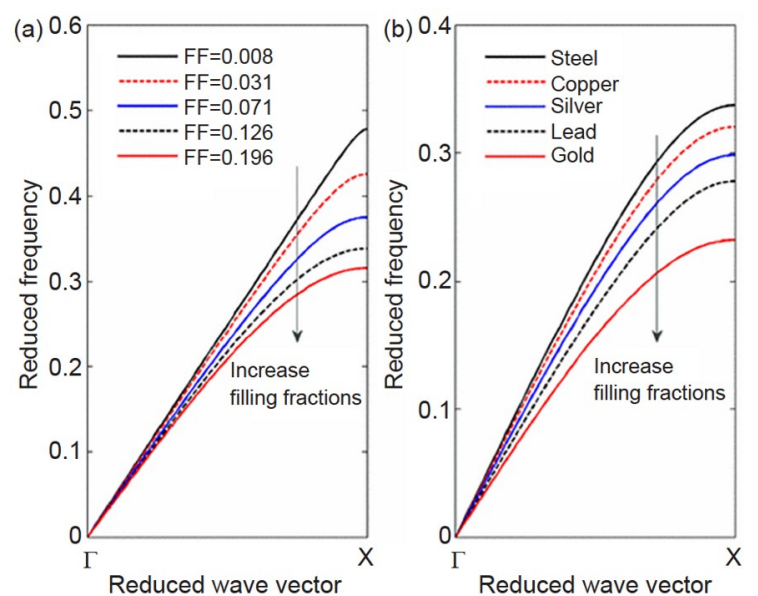

可以看出, 相速度不仅与弹性常数有关, 而且还与 平板的厚度 $h$ 有关．在没有任何散射体的情况下，通过 改变板的厚度分布可以设计出梯度折射率结构. 当所 有弹性常数保持不变时，弯曲波的折射率只与板厚度 相关, 通过改变板厚度可以设计各种透镜 ${ }^{[77 ~ 79]}$. 图4显 示了利用板厚度分布来构造Luneburg、Maxwell鱼 眼、90 ${ }^{\circ}$ 旋转、Eaton和聚焦透镜 ${ }^{[53,79]}$. 采用表 1 中的折 射率公式，其对应的厚度变化如图4(a)所示，高的折射 率对应较小厚度. 对于 $90^{\circ}$ 旋转透镜、Eaton透镜和聚光 透镜，透镜中心的最大折射率大于 5 ，相对厚度小于 $1 / 25$. 图4(b)显示了基于厚度变化所设计的几种透镜对 波的控制效果.

通过板厚度空间分布设计的透镜不仅可以应用于 波能量聚焦、波导方面的控制，也可用来解决波控制 中的隐身难题(声学斗篷). 声学斗篷的实现需要构造非 均匀性和各向异性材料, 但实际上制作起来相当复

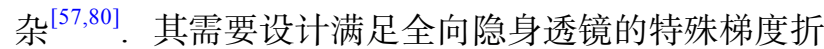
射率曲线, 该曲线要求中心附近的折射率为无穷大 ${ }^{[81]}$, 而由式(13)可以实现这种设计, 通过逐渐减小弹性板的 厚度使透镜中心的折射率接近极值. 在理想情况下, 板 的中心点厚度应为零，但是趋近于零的极小厚度足以 提供良好的隐身效果 ${ }^{[82]}$.

\section{3 梯度折射率透镜的应用}

利用梯度折射率透镜控制板内弹性波的传播，除 了基础的聚焦效果，还拓展了丰富的应用，如微/纳米
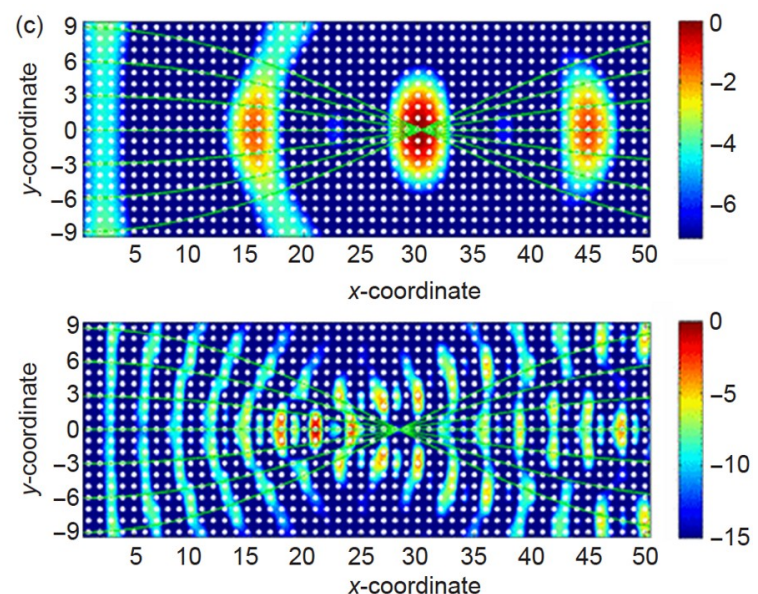

图 3 基于声子晶体能带特性的透镜设计 ${ }^{[8]}$. (a) 不同填充分数的钢/环氧声子晶体的一阶SV体声波模态能带图. (b) 不同固体填充材料的环氧声 子晶体一阶SV体声波模态能带图. (c) 有限差分模拟两种不同频率下SV波在声子晶体中的聚焦效果图

Figure 3 Lens design based on band characteristics of phononic crystals ${ }^{[8]}$. (a) Band diagrams of the first SV bulk acoustic mode for various filling fractions of a steel/epoxy phononic crystal. (b) Band diagrams of the first SV bulk acoustic mode for phononic crystals consisting of different solid cylinders in epoxy. (c) The finite difference time domain (FDTD) simulated SV wave focus in a phononic crystal at two different frequencies 
尺度上的波传播、热导率控制和光力学相互作用等, 这对物理、材料和纳米科学领域的研究具有意义.

Lin等人 ${ }^{[83]}$ 借助梯度折射率声子晶体的概念, 利用 声束宽压缩器将声能有效耦合到二维声子晶体波导中.
通过在声波传播的横向方向逐渐调制散射体的密度和 弹性模量，有效地将宽带声波压缩到声子晶体波导的 尺度, 可以应用于声生物传感器和信号处理器等. 图5(a) 显示了低阶反对称Lamb模态 $\left(\mathrm{A}_{0}\right)$ 在正梯度折射率透镜
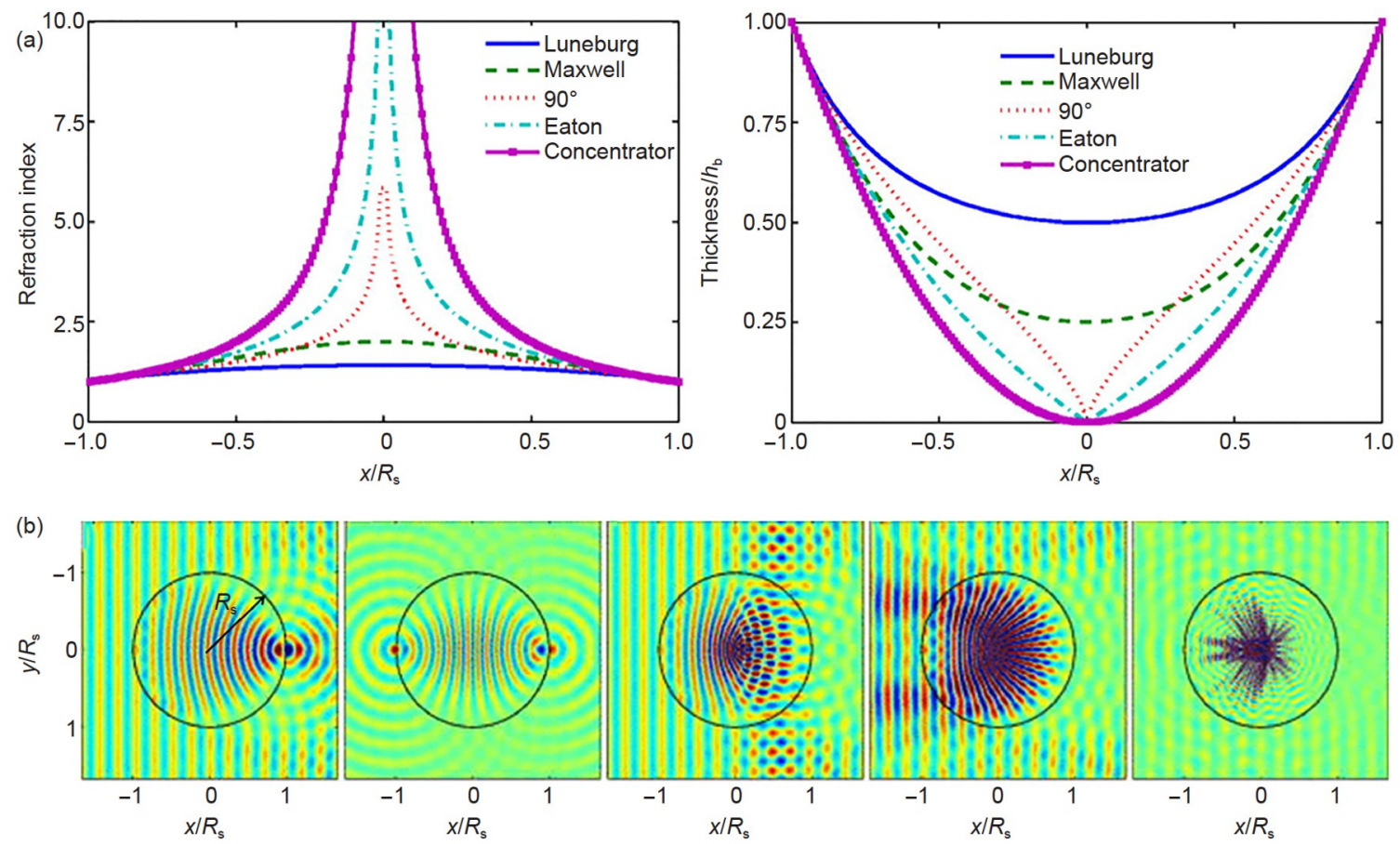

图 4 基于厚度变化的透镜设计. (a) 不同透镜折射率分布与厚度分布; (b) 几种透镜的波行为: Luneburg、Maxwell鱼眼、90旋转、Eaton和聚 焦透镜 ${ }^{[79]}$

Figure 4 Lens design based on thickness variation. (a) Refractive index and thickness distribution of different lenses. (b) Wave performance of different types of lenses: Luneburg, Maxwell's fish-eye, $90^{\circ}$ rotating, Eaton, and concentrator ${ }^{[79]}$
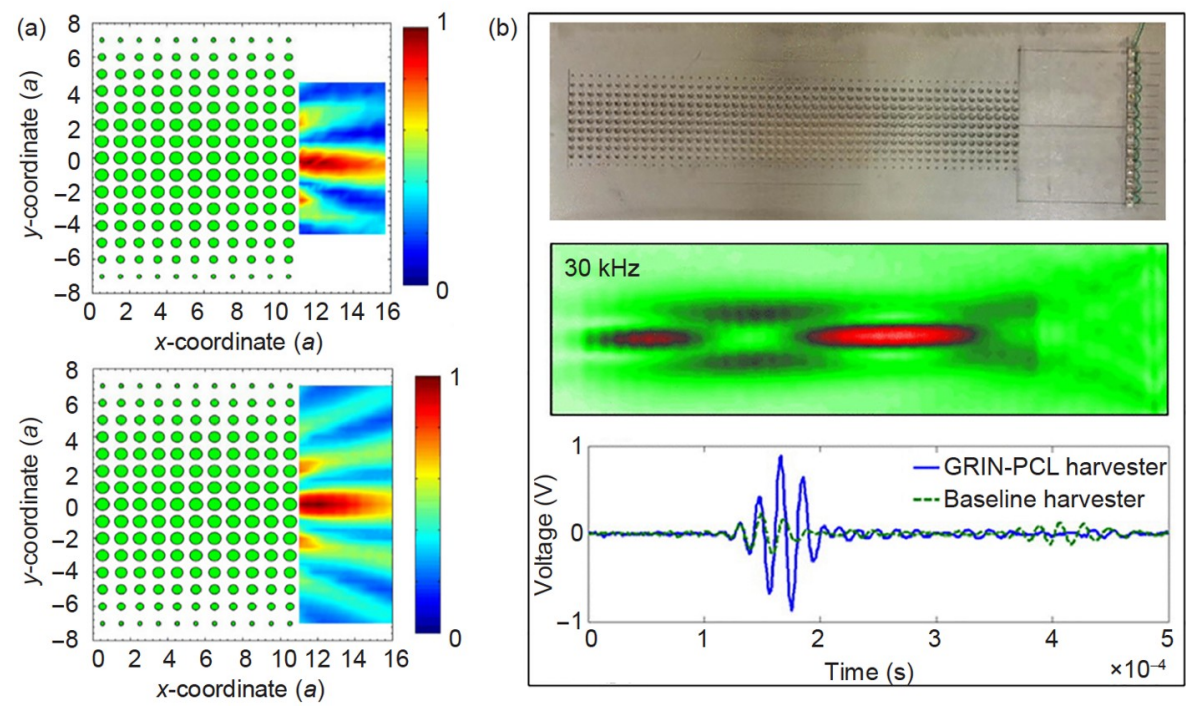

图 5 梯度折射透镜的应用. (a) 亚衍射聚焦的GRIN平面透镜 $(a \text { 表示单个正方形晶格的边长 })^{[84]}$; (b) GRIN平面透镜的能量采集 ${ }^{[85]}$

Figure 5 Applications of gradient refracting lenses. (a) A GRIN lens with sub-diffraction focusing ( $a$ is the length of a square lattice $)^{[84]}$; (b) a GRIN lens for energy harvesting ${ }^{[85]}$ 
后的聚焦现象. 通过分析空气/硅声子晶体柱的弯曲谐 振模式与振动模式耦合现象，发现谐振模式与平板振 动之间的偏振相干性所起的作用，从而帮助焦点包含 更好的信息，为声透镜超越衍射极限的性能作了展

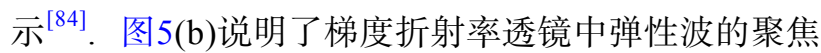

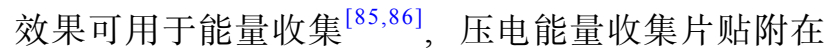
GRIN平面透镜背面，人射平面波被聚焦在一个点. 在 相同的平面波激励下，可以提取超过一个数量级的能 量输出.

梯度折射率声子晶体和超材料可以应用于各种类 型和不同频率的波，如低频的表面水波 ${ }^{[87,88]}$ 、频率在 几百到几万赫兹空气中的声波 ${ }^{[63,89 \sim 95]}$ 、几万到几十万 赫兹的水声声波 ${ }^{[66,97]}$ 以及固体中宽频带的瑞利波 ${ }^{[98 ~ 100]}$ 和兰姆波 ${ }^{[01 ~ 106]}$. 利用梯度透镜可以实现极致的波的控 制，包括聚焦 ${ }^{[8,63]}$ 、波导 ${ }^{[83,106]}$ 、偏折 ${ }^{[16,88,107]}$ 、隐 身 $^{[57,80 \sim 82]}$ 或能量收集 ${ }^{[55,86]}$ 等功能，其对波的操控能力 也被光学以外诸多学科如电子信息学 ${ }^{[108 ~ 110] 、}$ 、生物医 学 ${ }^{[111 ~ 113]}$ 、材料科学 ${ }^{[114,115]}$ 等广泛研究.

\section{4 总结与展望}

超材料的研究历史只有不到 20 年，但是其特殊设 计带来的负等效质量密度、负等效弹性模量、负折射
率等特性提供了许多在波动控制方面方便可行的方法. 梯度折射率超材料是一个操纵波的有效工具，其在光 学、声学、弹性波领域已取得一定的研究进展. 本文 对梯度折射率透镜的光学原理、3种在超材料领域的 实现方式和一些经典应用进行总结，希望为梯度折射 率超材料领域的研究提供有价值的参考.

相比传统光学透镜或者均质材料，梯度折射率超 材料制作的透镜在波导、聚焦、隐身等方面已经表现 出不凡的潜力. 但是, 目前还遇到诸多问题，例如：(1) 一些梯度折射率超材料的设计与最终呈现的结果还有 一定的差距，这与梯度透镜中的折射、衍射现象耦合 有关，这种偏差会导致工程应用方面出现问题. (2) 大 部分梯度透镜的设计在制作加工方面难度较大，而且 适用频率范围有限. 更有许多设计需要指定工作频率, 因此需要解决宽频适用的问题. (3) 纳米尺度的梯度折 射率透镜的研究还比较少, 但是纳米尺度材料和经典 物理学机制有所差异, 这是后续研究的机遇和挑战.

总之，目前梯度折射率超材料的设计方法和理论 原理已被广泛采纳和应用, 并取得了一定的成绩. 新的 设计方法和更多与工程实践相结合的应用还有待研究, 希望多尺度、跨学科等研究方式可以给这个领域以新 的创意与突破.

\section{参考文献}

1 Gomez-Reino C, Perez M V, Bao C. Gradient-index Optics: Fundamentals and Applications. Berlin: Springer Science \& Business Media, 2002

2 Centeno E, Cassagne D, Albert J. Mirage and superbending effect in two-dimensional graded photonic crystals. Phys Rev B, 2006, 73: 235119

3 Roux F S, de Leon I. Planar photonic crystal gradient index lens, simulated with a finite difference time domain method. Phys Rev B, 2006, 74: 113103

4 Cakmak A O, Colak E, Caglayan H, et al. High efficiency of graded index photonic crystal as an input coupler. J Appl Phys, 2009, 105: 103708

5 Torrent D, Sánchez-Dehesa J. Acoustic metamaterials for new two-dimensional sonic devices. New J Phys, 2007, 9: 323

6 Deng K, Ding Y, He Z, et al. Graded negative index lens with designable focal length by phononic crystal. J Phys D-Appl Phys, 2009, 42: 185505

7 Lin S S, Huang T J. Acoustic mirage in two-dimensional gradient-index phononic crystals. J Appl Phys, 2009, 106: 53529

8 Lin S C S, Huang T J, Sun J H, et al. Gradient-index phononic crystals. Phys Rev B, 2009, 79: 94302

9 Danawe H, Okudan G, Ozevin D, et al. Conformal gradient-index phononic crystal lens for ultrasonic wave focusing in pipe-like structures. Appl Phys Lett, 2020, 117: 21906

10 Allam A, Sabra K, Erturk A. Sound energy harvesting by leveraging a 3D-printed phononic crystal lens. Appl Phys Lett, 2021, 118: 103504

11 Walker E L, Reyes-Contreras D, Jin Y, et al. Tunable hybrid phononic crystal lens using thermo-acoustic polymers. ACS Omega, 2019, 4: 1658516590

12 Martin T P, Naify C J, Skerritt E A, et al. Transparent gradient-index lens for underwater sound based on phase advance. Phys Rev Appl, 2015, 4: 34003

13 Yan X, Zhu R, Huang G, et al. Focusing guided waves using surface bonded elastic metamaterials. Appl Phys Lett, 2013, 103: 121901

14 Wang W, Iglesias J, Jin Y, et al. Experimental realization of a pillared metasurface for flexural wave focusing. Apl Mater, 2021, 9: 51125

15 Cui X, Zhao J, Boyko O, et al. Multi-branch subwavelength focusing of the lowest-order antisymmetric lamb mode in a gradient-index phononic crystal. Int J Mech Sci, 2019, 157-158: 677-683

16 Zhang J, Zhang X, Xu F, et al. Vibration control of flexural waves in thin plates by 3D-printed metasurfaces. J Sound Vib, 2020, 481 : 115440 
17 Hyun J, Choi W, Kim M. Gradient-index phononic crystals for highly dense flexural energy harvesting. Appl Phys Lett, 2019, 115: 173901

18 Chen J, Zhao Y, Xing L, et al. Broadband bifunctional luneburg - Fisheye lens based on anisotropic metasurface. Sci Rep, 2020, 10: 20381

19 John S. Strong localization of photons in certain disordered dielectric superlattices. Phys Rev Lett, 1987, 58: 2486-2489

20 Yablonovitch E. Inhibited spontaneous emission in solid-state physics and electronics. Phys Rev Lett, 1987, 58: 2059-2062

21 Kushwaha M S, Halevi P, Dobrzynski L, et al. Acoustic band structure of periodic elastic composites. Phys Rev Lett, 1993, 71: 2022-2025

22 Sigalas M, Economou E N. Band structure of elastic waves in two dimensional systems. Solid State Commun, 1993, 86: 141-143

23 Zhu Z, Yang Y, Zhang N Z, et al. Locally resonant sonic materials. Physica B, 2000, 338: 201-205

24 Zhu R, Liu X N, Huang G L, et al. Microstructural design and experimental validation of elastic metamaterial plates with anisotropic mass density. Phys Rev B, 2012, 86: 144307

25 Li Y, Zhu L, Chen T. Plate-type elastic metamaterials for low-frequency broadband elastic wave attenuation. Ultrasonics, 2017, 73: 34-42

26 Sui N, Yan X, Huang T Y, et al. A lightweight yet sound-proof honeycomb acoustic metamaterial. Appl Phys Lett, 2015, 106: 171905

27 Fang N, Xi D, Xu J, et al. Ultrasonic metamaterials with negative modulus. Nat Mater, 2006, 5: 452-456

28 Jin Y, Bonello B, Moiseyenko R P, et al. Pillar-type acoustic metasurface. Phys Rev B, 2017, 96: 104311

29 Fuentes-Domínguez R, Yao M, Colombi A, et al. Design of a resonant luneburg lens for surface acoustic waves. Ultrasonics, 2021, 111: 106306

30 Danawe H, Okudan G, Ozevin D, et al. Conformal gradient-index phononic crystal lens for ultrasonic wave focusing in pipe-like structures. Appl Phys Lett, 2020, 117: 21906

31 Maxwell J C. Solutions of problems. Camb Dublin Math J, 1854, 8: 88

32 Houde-Walter S N, Moore D T. Delta- $n$ control in GRIN glass by additives in AgCl diffusion baths. Appl Opt, 1986, 25: 3373-3378

33 Moore D T. Gradient-index optics: A review. Appl Opt, 1980, 19: 1035-1038

34 Houde-Walter S. Lens designers: Gradient-index optics are in your future. Laser Focus World, 1989, 25: 151-160

35 Wood R W. Physical Optics. Stuttgart: The Macmillan Company Press, 1905

36 Luneburg R K. Mathematical Theory of Optics. Oakland: University of California Press, 1964

37 Stettler R. Über die optische abbildung von flächen und räumen. Optik, 1955, 12: 529-543

38 Morgan S P. General solution of the luneberg lens problem. J Appl Phys, 1958, 29: 1358-1368

39 Hamblen D P. Gradient refractive index optical lenses. USA Patent, US3486808A, 1969-12-30

40 Pearson A D, French W G, Rawson E G. Preparation of a light focusing glass rod by ion-exchange techniques. Appl Phys Lett, 1969, 15: 76-77

41 Siegman A E. Lasers. California: University Science Books Press, 1986

42 Wang S M, Zhao D M. Matrix Optics (in Chinese). Beijing: Higher Education Press, 2000 [王绍民, 赵道木. 矩阵光学. 北京: 高等教育出版社, 2000]

43 Alda J, Boreman G D. On-axis and off-axis propagation of gaussian beams in gradient index media. Appl Opt, 1990, 29: 2944-2950

44 Eaton J. On spherically symmetric lenses. In: Transactions of the IRE Professional Group on Antennas and Propagation. New York: IEEE, 1952. $66-71$

45 Rinehart R F. A solution of the problem of rapid scanning for radar antennae. J Appl Phys, 1948, 19: 860-862

46 Zickar M, Noell W, Marxer C, et al. Mems compatible micro-GRIN lenses for fiber to chip coupling of light. Opt Express, 2006, 14: 4237-4249

47 Mao Y, Chang S, Sherif S, et al. Graded-index fiber lens proposed for ultrasmall probes used in biomedical imaging. Appl Opt, 2007, 46: 58875894

48 Xie T, Guo S, Chen Z, et al. GRIN lens rod based probe for endoscopic spectral domain optical coherence tomography with fast dynamic focus tracking. Opt Express, 2006, 14: 3238-3246

49 Wang C, Ji N. Characterization and improvement of three-dimensional imaging performance of GRIN-lens-based two-photon fluorescence endomicroscopes with adaptive optics. Opt Express, 2013, 21: 27142-27154

50 Hajireza P, Shi W, Zemp R. Label-free in vivo GRIN-lens optical resolution photoacoustic micro-endoscopy. Laser Phys Lett, 2013, 10: 55603

51 Wang C, Mao Y X, Tang Z, et al. Numerical analysis of GRIN lens based miniature probes for optical coherence tomography (in Chinese). Opt Precis Eng, 2011, 19: 2300-2307 [王驰, 毛幼馨, 唐智, 等. 基于GRIN镜头的小型OCT探头的数值分析. 光学精密工程, 2011, 19: 2300-2307]

52 Huland D M, Charan K, Ouzounov D G, et al. Three-photon excited fluorescence imaging of unstained tissue using a GRIN lens endoscope. Biomed Opt Express, 2013, 4: 652-658

53 Aarbort M, Tyc T. Spherical media and geodesic lenses in geometrical optics. J Opt, 2012, 14: 75705

54 Martin T P, Nicholas M, Orris G J, et al. Sonic gradient index lens for aqueous applications. Appl Phys Lett, 2010, 97: 113503

55 Yi K, Collet M, Ichchou M, et al. Flexural waves focusing through shunted piezoelectric patches. Smart Mater Struct, 2016, 25: 75007

56 Ge Y, Sun H, Liu C, et al. Acoustic focusing by an array of heat sources in air. Appl Phys Express, 2016, 9: 66701

57 Cummer S A, Schurig D. One path to acoustic cloaking. New J Phys, 2007, 9: 45

58 Milton G W. The Theory of Composites. London: Cambridge University Press, 2002 
59 Torrent D, Håkansson A, Cervera F, et al. Homogenization of two-dimensional clusters of rigid rods in air. Phys Rev Lett, 2006, 96 : 204302

60 Torrent D, Sánchez-Dehesa J. Effective parameters of clusters of cylinders embedded in a nonviscous fluid or gas. Phys Rev B, 2006, 74: 224305

61 Berryman J G. Long-wavelength propagation in composite elastic media II. Ellipsoidal inclusions. J Acoust Soc Am, 1980, 68: 1820-1831

62 Cervera F, Sanchis L, Sánchez-Pérez J V, et al. Refractive acoustic devices for airborne sound. Phys Rev Lett, 2001, 88: 23902

63 Climente A, Torrent D, Sánchez-Dehesa J. Sound focusing by gradient index sonic lenses. Appl Phys Lett, 2010, 97 : 104103

64 Chang T M, Dupont G, Enoch S, et al. Enhanced control of light and sound trajectories with three-dimensional gradient index lenses. New J Phys, 2012, 14: 35011

65 Peng P, Xiao B, Wu Y. Flat acoustic lens by acoustic grating with curled slits. Phys Lett A, 2014, 378: 3389-3392

66 Titovich A S, Norris A N, Haberman M R. A high transmission broadband gradient index lens using elastic shell acoustic metamaterial elements. J Acoust Soc Am, 2016, 139: 3357-3364

67 Luo H, Shu W, Li F, et al. Anomalous wave propagation in quasiisotropic media. Opt Commun, 2006, 267: 271-277

68 Pendry J B. Negative refraction makes a perfect lens. Phys Rev Lett, 2000, 85: 3966-3969

69 Luo H, Ren Z, Shu W, et al. Construction of a polarization insensitive lens from a quasi-isotropic metamaterial slab. Phys Rev E, 2007, 75: 26601

70 Luo H, Ren Z, Shu W, et al. Construct a polarizing beam splitter by an anisotropic metamaterial slab. Appl Phys B, 2007, 87: 283-287

71 Qiu C, Zhang X, Liu Z. Far-field imaging of acoustic waves by a two-dimensional sonic crystal. Phys Rev B, 2005, 71: 54302

72 Tian Y, Tan Z, Han X, et al. Phononic crystal lens with an asymmetric scatterer. J Phys D-Appl Phys, 2018, 52: 25102

73 Tan Z, Wei Y, Tian Y, et al. Gradient negative refraction index phononic crystal lens with a rotating scatterer. Mater Res Express, 2019, 6: 96203

74 Allam A, Sabra K, Erturk A. Enhanced sound energy harvesting by leveraging gradient-index phononic crystals. In: ASME 2020 Conference on Smart Materials, Adaptive Structures and Intelligent Systems. New York: American Society of Mechanical Engineers, 2020. V001T07A004

75 Peng S, He Z, Jia H, et al. Acoustic far-field focusing effect for two-dimensional graded negative refractive-index sonic crystals. Appl Phys Lett, 2010, 96: 263502

76 Jin Y, Torrent D, Pennec Y, et al. Multimodal and omnidirectional beam splitters for lamb modes in elastic plates. AIP Adv, 2016, 6: 121602

77 Climente A, Torrent D, Sánchez-Dehesa J. Omnidirectional broadband insulating device for flexural waves in thin plates. J Appl Phys, 2013, 114: 214903

78 Zareei A, Darabi A, Leamy M J, et al. Continuous profile flexural GRIN lens: Focusing and harvesting flexural waves. Appl Phys Lett, 2018, 112: 23901

79 Climente A, Torrent D, Sánchez-Dehesa J. Gradient index lenses for flexural waves based on thickness variations. Appl Phys Lett, 2014, 105: 64101

80 Torrent D, Sánchez-Dehesa J. Acoustic cloaking in two dimensions: A feasible approach. New J Phys, 2008, 10: 63015

81 Miñano J C. Perfect imaging in a homogeneous three-dimensional region. Opt Express, 2006, 14: 9627-9635

82 Jin Y, Torrent D, Djafari-Rouhani B. Invisible omnidirectional lens for flexural waves in thin elastic plates. J Phys D-Appl Phys, 2017, 50: 225301

83 Lin S C S, Tittmann B R, Sun J H, et al. Acoustic beamwidth compressor using gradient-index phononic crystals. J Phys D-Appl Phys, 2009, 42: 185502

84 Zhao J, Bonello B, Boyko O. Focusing of the lowest-order antisymmetric lamb mode behind a gradient-index acoustic metalens with local resonators. Phys Rev B, 2016, 93: 174306

85 Tol S, Degertekin F L, Erturk A. Gradient-index phononic crystal lens-based enhancement of elastic wave energy harvesting. Appl Phys Lett, 2016, 109: 63902

86 Tol S, Degertekin F L, Erturk A. Phononic crystal luneburg lens for omnidirectional elastic wave focusing and energy harvesting. Appl Phys Lett, 2017, 111: 13503

87 Wang Z, Zhang P, Nie X, et al. Focusing of liquid surface waves by gradient index lens. EPL, 2014, 108: 24003

88 Wang Z, Zhang P, Nie X, et al. Manipulating water wave propagation via gradient index media. Sci Rep, 2015, 5: 16846

89 Park C M, Kim C H, Park H T, et al. Acoustic gradient-index lens using orifice-type metamaterial unit cells. Appl Phys Lett, 2016, 108: 124101

90 Wu L Y, Chen L W. An acoustic bending waveguide designed by graded sonic crystals. J Appl Phys, 2011, 110: 114507

91 Yuan B, Tian Y, Cheng Y, et al. An acoustic Maxwell's fish-eye lens based on gradient-index metamaterials. Chin Phys B, 2016, 25: 104301

92 Zigoneanu L, Popa B I, Cummer S A. Design and measurements of a broadband two-dimensional acoustic lens. Phys Rev B, 2011, 84: 24305

93 Wu L Y, Chen L W. Enhancing transmission efficiency of bending waveguide based on graded sonic crystals using antireflection structures. Appl Phys A, 2012, 107: 743-748

94 Zhu R, Ma C, Zheng B, et al. Bifunctional acoustic metamaterial lens designed with coordinate transformation. Appl Phys Lett, 2017, 110: 113503

95 Zhang Z, Li R Q, Liang B, et al. Controlling an acoustic wave with a cylindrically-symmetric gradient-index system. Chin Phys B, 2015, 24: 24301 
96 Su X, Norris A N, Cushing C W, et al. Broadband focusing of underwater sound using a transparent pentamode lens. J Acoust Soc Am, 2017, 141: $4408-4417$

97 Zhao S, Wang Y, Zhang C. High-transmission acoustic self-focusing and directional cloaking in a graded perforated metal slab. Sci Rep, 2017, 7: 4368

98 Colombi A, Ageeva V, Smith R J, et al. Enhanced sensing and conversion of ultrasonic Rayleigh waves by elastic metasurfaces. Sci Rep, 2017, 7: $1-9$

99 Zhao J, Bonello B, Becerra L, et al. Focusing of rayleigh waves with gradient-index phononic crystals. Appl Phys Lett, 2016, 108: 221905

100 Sun J, Yu Y. Beam focusing of surface acoustic wave using gradient-index phononic crystals. In: IEEE International Ultrasonics Symposium. New York: IEEE, 2016

101 Wang D F, Wang Y H, Chuang K C. Nearly-isotropic adjustable phononic crystal lenses using concentrated balls with hertz contacts. Phys Lett A, 2021, 396: 127240

102 Jin Y, Torrent D, Pennec Y, et al. Gradient index devices for the full control of elastic waves in plates. Sci Rep, 2016, 6: 24437

103 Zhao J, Marchal R, Bonello B, et al. Efficient focalization of antisymmetric lamb waves in gradient-index phononic crystal plates. Appl Phys Lett, 2012, 101: 261905

104 Hyun J, Cho W H, Park C S, et al. Achromatic acoustic gradient-index phononic crystal lens for broadband focusing. Appl Phys Lett, 2020, 116: 234102

105 Zhao J, Bonello B, Boyko O. Beam paths of flexural lamb waves at high frequency in the first band within phononic crystal-based acoustic lenses. AIP Adv, 2014, 4: 124204

106 Wu T T, Chen Y T, Sun J H, et al. Focusing of the lowest antisymmetric lamb wave in a gradient-index phononic crystal plate. Appl Phys Lett, 2011, 98: 171911

107 Torrent D, Pennec Y, Djafari-Rouhani B. Omnidirectional refractive devices for flexural waves based on graded phononic crystals. J Appl Phys, 2014, 116: 224902

108 Acosta L K, Bertó-Roselló F, Xifre-Perez E, et al. Stacked nanoporous anodic alumina gradient-index filters with tunable multispectral photonic stopbands as sensing platforms. ACS Appl Mater Interfaces, 2018, 11: 3360-3371

109 Liu Y, Li S, Guo J, et al. Planar microwave retroreflector based on transmissive gradient index metasurface. New J Phys, 2020, 22: 63044

110 Ganapathy B, Krishnaswamy S C. Energy transfer using gradient index metamaterial. Int J Antennas Propag, 2018, 2018: 7946168

111 Accanto N, Chen I W, Ronzitti E, et al. Multiplexed temporally focused light shaping through a gradient index lens for precise in-depth optogenetic photostimulation. Sci Rep, 2019, 9: 7603

112 Liu L, Shi H, Liu X. Adaptability analysis of radiative transport diffusion approximation in planar-graded-index media. Adv Mech Eng, 2018, 10: 1687814018809613

113 Reynolds T A, Jensen A R, Bellairs E E, et al. Dose gradient index for stereotactic radiosurgery/radiation therapy. Int J Radiat Oncol Biol Phys, 2020, 106: 604-611

114 Lim S Y, Law C S, Markovic M, et al. Engineering the slow photon effect in photoactive nanoporous anodic alumina gradient-index filters for photocatalysis. ACS Appl Mater Interfaces, 2018, 10: 24124-24136

115 Forestier X, Karpate T, Huss G, et al. Supercontinuum generation in nanostructured core gradient index fibers. App1 Nanosci, 2020, 10: 19972005 


\title{
A review of gradient index metamaterials lenses
}

\author{
Danfeng Wang, Zhiyuan Ren \& Kuo-Chih Chuang* \\ Institute of Applied Mechanics, School of Aeronautics and Astronautics, Zhejiang University, Hangzhou 310058, China \\ * Corresponding author, E-mail: chuangkc@zju.edu.cn
}

As a kind of vibration propagation phenomenon existing in nature, waves have been captured and studied by people for a long time. With the concept of metamaterials proposed in recent decades, the manipulation and regulation of waves have reached a new height. Metamaterials are composites with artificially designed structures and supernormal physical properties that natural materials do not have. Generally, most of them have special characteristics such as negative equivalent mass densities, negative equivalent elastic modulus, negative refractive index, and so on. As a kind of metamaterials, phononic crystals are periodic structures whose Bragg band gaps or local resonant band gaps forbid the propagation of sound waves or elastic waves. The band characteristics can be adjusted by elaborate design, so that the acoustic waves or elastic waves under the passband frequency can achieve extreme control functions of wave propagation such as imaging, focusing and directional transmission.

Gradient-index metamaterials are composites, whose refractive index varies with space, and have been employed in various applications. Gradient index lenses are composed of locally inhomogeneous materials, in which the refractive index is a function of spatial coordinates and the waves propagate along the curved trajectory. After proper design, they can have the functions of bending, deflection or focusing of the waves. The core of the design is to distribute the effective refractive index of the lens reasonably, which can be changed locally by changing the properties of each cell, such as the lattice size of phononic crystals, the filling rate of scatters, the material of scatters, and so on. In addition, Gradient index metamaterials can be designed to accurately focus waves over a wide frequency range for several engineering applications such as traveling wave energy harvesting for self-powered electronic devices.

In this review, we firstly introduce the concept of gradient lens in optics and its history during about two hundred years. The relationship between refractive index distribution and the trajectory of wave propagation is analyzed theoretically. The typical hyperbolic refraction trajectories are listed in detail. The refractive index distribution formulas and focusing schematic diagram of several commonly known lenses (Luneburg, Eaton, etc.) are given. Then, three design methods of gradient index lens are introduced. First of them is the equivalent medium method, which helps to deduce the equivalent material parameters for the calculation of the effective speed of waves. Secondly, some research is based on the band characteristics of phononic crystals. The final method is using the size distribution of elastic plates to design structures with gradient index. Each method is introduced not only in theory but also in cases. In addition, we also introduce several research of applying gradient lenses to wave control. Finally, the conclusions and drawbacks of the above gradient index metamaterials are summarized. It is pointed out that although the present lens designs have achieved some encouraging results, they are still confronted with several key problems such as the realizations of low-frequency and broadband, nanoscale, minor-error, lightweight and the manufacture and testing of samples, and the environmental adaptations requirements. Therefore, there is still a long way for the current gradient index metamaterials toward the real application. The trend and outlook toward future are also presented. It is hoped that this review could provide guidelines for the design and realization of gradient index lenses.

gradient index, lens, equivalent media, phononic crystals, metamaterials

doi: 10.1360/TB-2021-0523 